\title{
How to estimate distance and velocity from parallax and proper motion
}

\author{
Andrei P. Igoshev and Frank Verbunt and Eric Cator \\ IMAPP Radboud University Nijmegen \\ P.O. Box 90106500 GL Nijmegen The Netherlands \\ email: ignotur@gmail.com
}

\begin{abstract}
If the observed parallax $\varpi^{\prime}$ has a gaussian measurement error $\sigma$, there is a $68 \%$ probability that the actual parallax $\varpi$ is in the range $\varpi^{\prime}-\sigma<\varpi<\varpi^{\prime}+\sigma$ (the frequentist approach). The probability distribution within this range is not known from $\varpi^{\prime}$ and $\sigma$ alone, and in particular, we cannot state that the most probable distance $D$ is given by $D=1 / \varpi^{\prime}$. To obtain a probability distribution, we need to know or assume a distribution of pulsar distances. Similar assumptions are also required to estimate the velocity distribution of radio pulsars.
\end{abstract}

Keywords. methods: statistical, stars: distances

\section{Conditional probability}

For a detailed discussion of the conversion of the parallax to distance and of the role of priors, we refer to an excellent paper by Bailer-Jones (2015). Here we briefly summarize the articles of Igoshev et al. (2016) and of Verbunt et al. (2017).

If an object with a real parallax $\varpi$ and distance $D=1 / \varpi$ is measured with a gaussian measurement error $\sigma$, the probability of measuring $\varpi^{\prime}$ when the real value is $\varpi$ is

$$
p\left(\varpi^{\prime} \mid \varpi\right) d \varpi^{\prime}=p\left(\varpi^{\prime} \mid 1 / D\right) d \varpi^{\prime}=\frac{1}{\sigma \sqrt{2 \pi}} \exp \left[-\frac{\left(1 / D-\varpi^{\prime}\right)^{2}}{2 \sigma^{2}}\right] d \varpi^{\prime} .
$$

In this equation $\varpi^{\prime}$ varies and $\varpi$ is fixed. There is an approximately $68 \%$ probability that the measured parallax $\varpi^{\prime}$ lies in the range $\left|\varpi^{\prime}-\varpi\right|<\sigma$, or equivalently that $\varpi^{\prime}-\sigma<\varpi<\varpi^{\prime}+\sigma$. However, a measurement $\varpi^{\prime}$ may also result from a different $\varpi_{2} \neq \varpi$. In that case there is still a probability of $68 \%$ that $\varpi^{\prime}-\sigma<\varpi_{2}<\varpi^{\prime}+\sigma$. Therefore, from $\varpi^{\prime}$ and $\sigma$ we can indicate an interval for the actual parallax $\varpi$ with a corresponding probability, but not the probability distribution within or outside the interval. This is the frequentist approach.

To obtain a probability distribution, we need to know or assume a prior probability distribution of the parallaxes; in practice a prior of the distance distribution, $f(D)$, is used. This prior acts as a weighting, and for the probability of a real distance $D$ for a fixed measurement $\varpi^{\prime}$ we have

$$
p\left(D \mid \varpi^{\prime}\right) d D=C f(D) \frac{1}{\sigma \sqrt{2 \pi}} \exp \left[-\frac{\left(1 / D-\varpi^{\prime}\right)^{2}}{2 \sigma^{2}}\right] d D
$$

where $C$ is a normalization constant.

Various authors, e.g. Verbiest et al.(2012), following Faucher-Giguère \& Kaspi (2006), erroneously replace $d D$ in Eq.(1.2) with $d \varpi=\left(1 / D^{2}\right) d D$, which leads to a wrong weighting of $D$. 


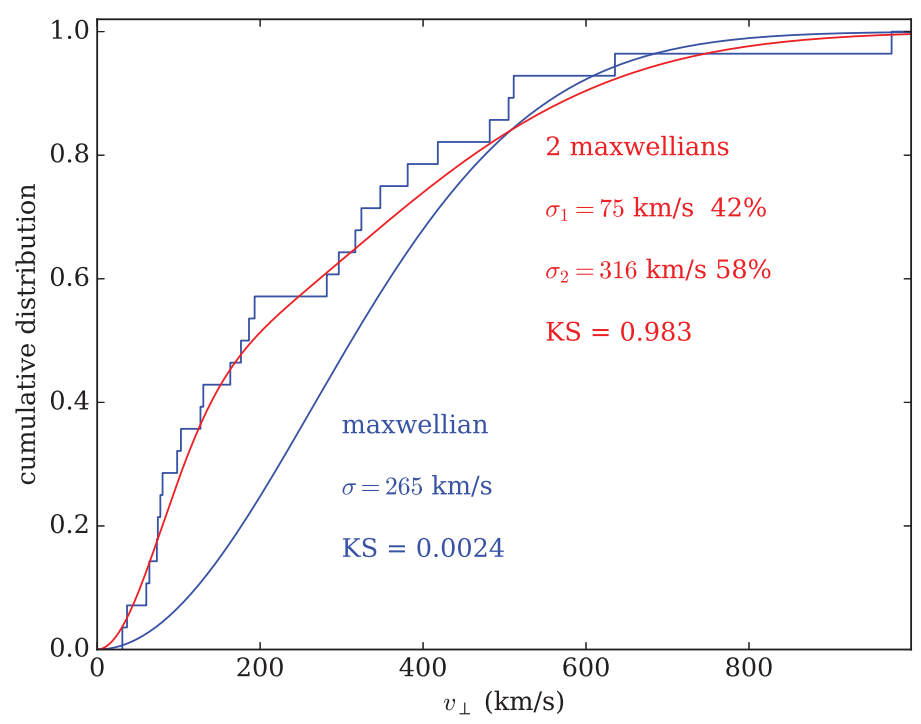

Figure 1. Our best model velocity distribution, the sum of two Maxwellians, converts to a good description (red line) of the observed cumulative distribution of nominal projected pulsar velocities $v_{\perp}^{\prime} \equiv \mu^{\prime} / \varpi^{\prime}$ (histogram), in contrast to the single Maxwellian found by Hobbs et al. (2005, blue line). The $p$-values of one-sided Kolmogorov-Smirnov tests confirm this.

\section{Velocities of young radio pulsars}

The measured velocity projected on the sky is found by combining a measured parallax and a measured proper motion: $v_{\perp}^{\prime}=\mu^{\prime} / \varpi^{\prime}$. Thus each model velocity must be converted into a parallax and proper motion to properly take into account the measurement errors in the model fitting. This requires a (known or assumed) distance distribution $f(D)$.

We have found that the bimodal distribution which consists of two maxwellians with $\sigma_{1}=75_{-15}^{+20} \mathrm{~km} / \mathrm{s} \sigma_{2}=316_{-40}^{+58} \mathrm{~km} / \mathrm{s}$ and $w=0.42_{-0.12}^{+0.10}$ describes the young isolated radio pulsar velocity distribution much better than the single maxwellian with $\sigma=265 \mathrm{~km} / \mathrm{s}$ that describes the result from a non-parametric analysis by Hobbs et al. (2005). A direct comparison of the velocity distribution with the nominal pulsar velocities $v_{\perp}^{\prime}=\mu^{\prime} / \varpi^{\prime}$, illustrates this well (Figure 1).

\section{References}

Bailer-Jones, C. A. L. 2015, PASP, 127, 994

Faucher-Giguère, C.-A. \& Kaspi, V. M. 2006, ApJ, 643, 332

Hobbs G., Lorimer, D. R., Lyne A. G., \& Kramer M. 2005, MNRAS, 360, 3

Igoshev A. \& Verbunt, F. Cator, E. 2016, A\& A, 591, A123

Verbiest J. P. W., Weisberg J. M., Chael A. A., Lee K. J., \& Lorimer D. R. 2012, ApJ, 755, 39

Verbunt F., Igoshev A., Cator E. 2017, AESA in press ArXiv: 1708.08281 